\title{
Intensive nursing work schedules and the risk of hypoglycaemia in critically ill patients who are receiving intravenous insulin
}

\author{
Kimberley Louie, ${ }^{1}$ Rupi Cheema, ${ }^{2}$ Peter Dodek, ${ }^{1,3,4}$ Hubert Wong, ${ }^{4}$ Amanda Wilmer, ${ }^{1}$ \\ Maja Grubisic, ${ }^{4}$ John Mark FitzGerald, ${ }^{5}$ Najib T Ayas, ${ }^{1,2,3,5}$
}

\begin{abstract}
- An appendix is published online only. To view this file please visit the journal online (http://qshc.bmj.com).

${ }^{1}$ Program in Critical Care Medicine, Providence Health Care, University of British Columbia, Vancouver, Canada ${ }^{2}$ Sleep Disorders Program, University of British Columbia Hospital, Vancouver, Canada ${ }^{3}$ Critical Care Division, Department of Medicine, University of British Columbia, Vancouver, Canada ${ }^{4}$ Center for Health Evaluation and Outcome Sciences, Providence Health Care, Vancouver, Canada ${ }^{5}$ Centre for Clinical Epidemiology and Evaluation, Vancouver Coastal Health Research Institute, Vancouver, Canada
\end{abstract}

\section{Correspondence to}

Dr Najib Ayas, Room 224 Comox Building, 1081 Burrard Street, Vancouver, BC V6Z 1Y6 Canada;

nayas@providencehealth.bc.ca

Accepted 19 December 2009 Published Online First 4 August 2010

\begin{abstract}
Rationale Nurses in the intensive care unit (ICU) commonly work frequent $12 \mathrm{~h}$ shifts, potentially leading to fatigue and reduced vigilance. The authors hypothesised that rates of hypoglycaemia in patients receiving an insulin infusion would be associated with the intensity of work of the bedside nurse in the preceding $72 \mathrm{~h}$.

Methods The authors identified ICU patients who had hypoglycaemia (glucose $\leq 3.5 \mathrm{mmol} / \mathrm{l}, 63 \mathrm{mg} / \mathrm{dl}$ ) between October 2006 and June 2007. The number of shifts worked in the previous $72 \mathrm{~h}$ was calculated for the nurse caring for the patient when the event occurred (case shift). For each case shift, the authors identified up to three control shifts $(24,48$ and $72 \mathrm{~h}$ before the event in the same patient) and calculated the number of shifts worked by nurses on these shifts in the previous $72 \mathrm{~h}$. Conditional logistic regression was used to determine whether the number of shifts worked was associated with hypoglycaemia.
\end{abstract}

Results There were 41 events (32 patients). Each additional shift worked in the previous $72 \mathrm{~h}$ was associated with a significantly increased risk of hypoglycaemia $(\mathrm{OR}=1.65 /$ shift, $95 \% \mathrm{Cl} 1.01$ to 2.68, $p=0.04)$ after controlling for nurse age and experience. The association was greater for the 23 events associated with an error in management according to the insulin protocol $(\mathrm{OR}=2.93 /$ shift, 1.15 to $7.44, \mathrm{p}=0.02)$ compared with events not associated with an error $(O R=1.34 /$ shift, 0.73 to $2.45, p=0.34)$.

Conclusions Intensive nursing work schedules are associated with hypoglycaemic events in ICU patients.

\section{INTRODUCTION}

Patients in the intensive care unit (ICU) have complex medical problems, have little physiological reserve, are subjected to numerous risky medications and have high rates of medical errors. ${ }^{1} \mathrm{~A}$ number of safety outcomes are of particular concern in ICU patients, as they occur more commonly (or sometimes exclusively) in ICU settings, are potentially preventable and are associated with considerable morbidity and mortality. ${ }^{2}$ One such safety outcome is hypoglycaemia while receiving intravenous insulin infusions. ${ }^{3}$

Although the exact level of glucose control required in ICU patients is unclear, ${ }^{45}$ most would agree that blood glucose concentration should not be allowed to be substantially elevated above normal. Because of stress, medications and diabetes, many patients in the ICU require infusions of insulin to maintain blood glucose concentration in an acceptable range. ${ }^{6}$ The major side effect of insulin infusions is hypoglycaemia, which may be associated with confusion, seizures and neurological damage. Furthermore, hypoglycaemic events are an independent risk factor for mortality. ${ }^{7}$

In the ICU, first-line patient care is provided by nurses who are specially trained in critical care. They must be knowledgeable of complex medical problems and the management of risky intravenous medication infusions. Furthermore, they must be alert to subtle changes in their patient's condition, as patients may deteriorate abruptly with little warning. There is currently a shortage of ICU nurses leading to demanding work schedules. These schedules result in acute and chronic sleep deprivation, which may compromise vigilance. ${ }^{8}$

The purpose of this study was to assess the relationship between intensive nursing work schedules and hypoglycaemia in ICU patients receiving intravenous insulin infusions. Specifically, we determined whether the number of $12 \mathrm{~h}$ shifts worked by the bedside nurse in the $72 \mathrm{~h}$ antecedent to their work shift was associated with the occurrence of hypoglycaemia.

\section{METHODS}

\section{Setting and participants}

Between 6 October 2006 and 30 June 2007, we collected data from patients admitted to the ICU at St Paul's Hospital (SPH) in Vancouver, British Columbia. This ICU is a 15 -bed medical-surgical unit within a 400-bed tertiary care hospital. The ICU is staffed by rotating residents, fellows and dedicated intensivists. Because of high patient acuity, the nurse:patient ratio is generally $1: 1$ (about $85 \%$ of patients).

All nurses in the ICU work $12 \mathrm{~h}$ shifts $(7.30 \mathrm{am}$ to $7.30 \mathrm{pm}$ ) with patient 'handoff' reports occurring from 7.30 to $7: 45 \mathrm{am}$ and from 7.30 to $7: 45$ $\mathrm{pm}$. The mean age of nurses who work in the ICU is 40.9 years, and approximately $70 \%$ have at least 2 years of experience. In contrast to many other ICUs, travelling nurses and nurses from agencies do not work in our ICU. When additional nurses are necessary because of an unscheduled absence (eg, illness) or because of patient workload, the charge nurse will contact off-duty nurses to determine if any of them is available to work overtime. The contacted nurses are not coerced or mandated to work extra shifts.

For all patients in the ICU (other than diabetic ketoacidosis) requiring intravenous insulin to control 
hyperglycaemia, nurses are strongly encouraged to manage infusions according to a protocol that was developed by the SPH ICU pharmacist and physicians (see online Appendix). In general, the infusions are prepared by Pharmacy and sent to the ICU.

The study obtained ethical approval from the Institutional Review Board of the University of British Columbia.

\section{Ascertainment of hypoglycaemic events}

As part of ongoing data collection for a clinical ICU database, each day a research nurse reviewed patient charts and recorded detailed information regarding demographics, diagnoses, severity of illness, procedures, complications and adverse safety outcomes (including the occurrence of hypoglycaemia, defined as a blood glucose concentration $\leq 3.5 \mathrm{mmol} / 1 \quad(63 \mathrm{mg} / \mathrm{dl})^{9}$ by bedside fingerstick or serum measurement). This information was entered by specially trained ICU nurses who reviewed all charts concurrently and were unaware of the hypotheses for this study. We did not have any detailed information concerning symptoms or signs of hypoglycaemia, as these are not captured routinely.

For this analysis, we included only hypoglycaemic events that occurred when the patient was on an intravenous insulin infusion. Multiple events in a single patient were included. However, we excluded events that occurred within 3 days of a previous event because the occurrence of one hypoglycaemic event could modify the risk of further events in the short term (eg, subsequent nurses caring for the patient may become more vigilant), and we did not want case and control shifts to overlap.

For each event, one of the investigators (KL) reviewed the patient chart to confirm that hypoglycaemia occurred, and that the patient was receiving an intravenous insulin infusion at the time of the event. Furthermore, we ascertained whether the nurse caring for the patient made an error in management (ie, insulin dose or blood sugar monitoring deviated from the protocol, or insulin dose not decreased after tube feedings stopped) that may have contributed to the event.

\section{Collection of work schedule information}

Each day, patient flowsheets were reviewed by a separate research nurse to identify the specific bedside nurse who took care of each patient during the day and night shifts; nurses were identified only as a unique code number in a confidential database. The nurse who signed the flowsheet for the majority of the shift (done every hour) was considered the bedside nurse. For each nurse, we also obtained their age and years of experience in the ICU at SPH.

\section{Identification of case shifts}

For each hypoglycaemic event, the nurse taking care of the patient during the shift of the event (case shift) was identified. The number of shifts worked by this nurse in the $72 \mathrm{~h}$ before starting the case shift was calculated (including shifts in the hospital outside the ICU). Because of a potential lag between infusion errors and hypoglycaemia, events occurring between 7.30 and 8.30 am and between 7.30 and 8.30 pm were attributed to the nurse working the preceding shift.

\section{Identification of control shifts}

For each case shift, we identified up to three control shifts. Specifically, control shifts were the work shifts 24,48 and $72 \mathrm{~h}$ before the hypoglycaemic event in the same patient during which the patient was receiving an intravenous infusion of insulin in the ICU. For each control shift, the number of shifts worked by the bedside nurse during the previous $72 \mathrm{~h}$ was calculated.

\section{STATISTICAL ANALYSIS}

We used a within-patients analysis to control for patient confounders (APACHE II, admitting diagnosis, etc). The effect of the number of shifts worked on the incidence of hypoglycaemia was assessed using conditional logistic regression with all event and control shifts for each patient treated as a cluster. ${ }^{10}$ This analysis controlled for multiple events in a single patient. To adjust for potential confounding by nurse attributes, the conditional logistic regression analysis was repeated, including the nurse age and ICU experience (dichotomised as $\geq 2$ years vs $<2$ years of experience) in the model.

Results are expressed as mean \pm SD unless otherwise indicated. A $p$ value of $<0.05$ was considered statistically significant.

\section{RESULTS}

\section{Description of case and control shifts}

A total of 55 hypoglycaemic events were identified in 36 patients. Ten events were excluded, as they occurred within 3 days of a preceding event, and four events were excluded, because there was no suitable control period before the event (patient was not on an insulin infusion 24 or more hours before the event). This left a total of 41 events in 32 patients. The mean age of the patients was $59.5 \pm 15.6$ years, and the mean APACHE II Score was $28.2 \pm 8.7$. The most common primary admitting diagnoses included: sepsis/septic shock (17 patients), COPD exacerbation (11 patients) and ARDS (seven patients).

The events were fairly evenly distributed among the nurses; 34 different nurses worked during the 41 events (ie, 28 nurses worked during one event, five worked during two events, and one nurse worked during three events).

The distribution of the hypoglycaemic events according to time of day is shown in figure 1. Six events occurred at transition time periods (five from 7.30 to 8.30 am and one from 7.30 to $8.30 \mathrm{pm})$.

Ninety-two control shifts matched to the 41 case shifts were identified (mean of 2.24 control shifts per case shift). Characteristics of the nurses who worked the case and control shifts, and the distribution of the number of shifts worked by these nurses in the previous $72 \mathrm{~h}$ are shown in table 1 . None of the nurses worked consecutive (double) shifts. During essentially all of the shifts (97\%), the nurse was primarily responsible for one patient (ie, 1:1 nurse:patient ratio). The mean number of shifts worked in the antecedent $72 \mathrm{~h}$ by the nurses who worked during a case shift was greater than those who worked during the control shifts (1.29 vs 1.07 shifts).

\section{Main analysis}

Conditional logistic regression (table 2) showed that each additional shift worked in the previous $72 \mathrm{~h}$ was significantly associated with an increased incidence of hypoglycaemia ( $\mathrm{OR}=1.56$ per shift, $95 \%$ CI 0.98 to $2.48, \mathrm{p}=0.05$ ).

Inclusion of nurse age and ICU experience in the conditional regression analysis did not change the OR appreciably (1.65; table 2). Of note, neither age nor ICU experience was significantly associated with the occurrence of hypoglycaemia. We did not include nurse:patient ratio as a covariate, as essentially all the shifts $(97 \%)$ had a ratio of $1: 1$ (table 1$)$.

\section{Secondary analyses}

The adjusted OR were similar for day and night shifts. For events that occurred during night shifts, the adjusted OR associated with each additional shift worked in the previous $72 \mathrm{~h}$ was 2.32 (95\% CI 1.02 to 5.25), while for events that occurred during day shifts, the OR was 1.64 (0.77 to 3.45). 

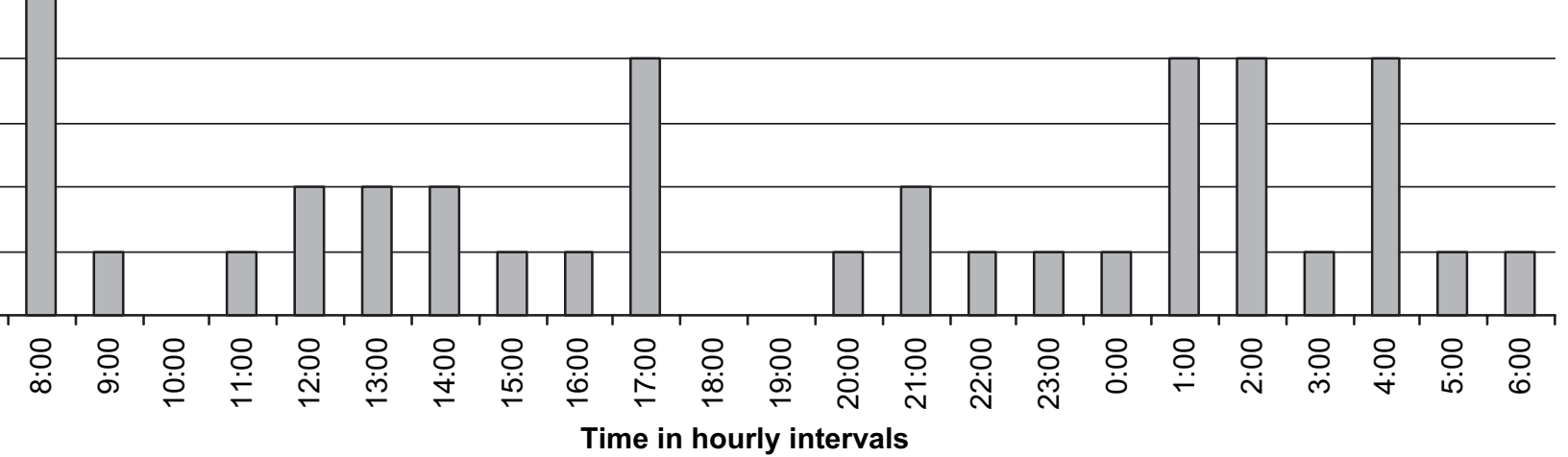

Figure 1 Distribution of hypoglycaemic events by time of day.

We stratified the analysis by events associated with an error in management according to the insulin protocol (table 3). In 23 $(56 \%)$ of the events, an error in management according to the insulin protocol was associated with the event. Nineteen were considered to be an error due to inadequate monitoring of blood sugar, and four an error in infusion rate modification. For the 23 events associated with an error, there were 46 control shifts. In the adjusted analysis, each additional shift worked was associated with a markedly increased $\mathrm{OR}$ of an event $(\mathrm{OR}=2.93,95 \%$ CI 1.15 to $7.44, p=0.024$ ). For the 18 events not associated with an error, the OR was much less (1.34) and not statistically significant ( $95 \%$ CI 0.73 to $2.45, \mathrm{p}=0.34$ ).

\section{DISCUSSION}

In this single-centre study, the increased number of antecedent shifts worked by bedside nurses was associated with an increased incidence of hypoglycaemia. Each additional shift increased the odds of an event occurring by 1.65 after controlling for nurse age and ICU experience. The strength of association was greater when the events were associated with an error in management compared with events where no error was identified.

We propose that the increased frequency of hypoglycaemia was related to the effects of fatigue of the bedside nurse. Physiological studies have demonstrated substantial adverse cognitive effects of even moderate degrees of chronic sleep deprivation. After 2 weeks of spending $6 \mathrm{~h}$ in bed per night, cognitive performance was similar to that after $24 \mathrm{~h}$ without sleep; ${ }^{11}$ this is of concern, as psychomotor performance after $24 \mathrm{~h}$ of continuous wakefulness is similar to that in the presence of a blood alcohol concentration of $0.10 \%$ (legally intoxicated) ${ }^{12}$

Table 1 Characteristics of case and control shifts

\begin{tabular}{lll}
\hline & Case shifts (N=41) & Control shifts (N=92) \\
\hline Age of nurse (SD) & $40.95(8.69)$ & $40.35(5.82)$ \\
$\begin{array}{l}\text { Mean no of shifts 72 } \mathrm{h} \\
\text { antecedent to index shift }\end{array}$ & 1.29 & 1.07 \\
No (\%) who worked 0, 1, 2, 3 in previous $72 \mathrm{~h}$ & \\
0 shifts & $9(22.0 \%)$ & $31(33.7 \%)$ \\
1 shift & $15(36.6)$ & $33(35.9)$ \\
2 shifts & $13(31.7)$ & $19(20.7)$ \\
3 shifts & $4(9.76)$ & $9(9.78)$ \\
$\begin{array}{l}\text { No (\%) who had } \geq 2 \text { years } \\
\text { intensive care unit experience }\end{array}$ & $27(66 \%)$ & $51(55 \%)$ \\
$\begin{array}{l}\text { No (\%) of shifts with 1:1 } \\
\text { nurse:patient ratio }\end{array}$ & $40(98 \%)$ & $90(98 \%)$ \\
\hline
\end{tabular}

Also, rates of industrial incidents (accidents and injuries) in nonhealthcare industries increase with successive night shifts. On average, compared with the first night shift, incidents are $6 \%$ more likely on the second night, $17 \%$ more likely on the third night and $36 \%$ more likely on the fourth night shift. ${ }^{13}$ Studies using simulated healthcare tasks under controlled conditions have also demonstrated substantial effects of sleep deprivation. Parshuram and colleagues studied predictors of errors in preparation of narcotic infusions in 118 healthcare workers (81 of whom were nurses). Less sleep in the previous $24 \mathrm{~h}$ was significantly $(p=0.02)$ associated with the occurrence of large errors in medication preparation. ${ }^{14}$

Working frequent $12 \mathrm{~h}$ shifts (especially on multiple consecutive days) leaves little time for adequate sleep between shifts, especially when the time required for commuting and patient 'hand-offs' at the beginning and end of work shifts are considered. A recent study has shown that nurses obtain only $6.5 \mathrm{~h}$ of sleep between $12 \mathrm{~h}$ work shifts, ${ }^{8}$ an amount insufficient to maintain optimal cognitive performance. ${ }^{15}$

Our results extend those of other investigators who have studied the impact of nursing work schedules on patient safety. Sixty-five per cent of nurses who completed 14-day log books reported struggling to stay awake at work at least once during the study period, and $27 \%$ reported making at least one error, the majority of which involved administration of medications (including insulin and anticoagulants). Error rates increased as duration of shifts increased ( $p=0.03$ ); $2 \%$ of shifts $\leq 8.5 \mathrm{~h}$ in length were associated with at least one self-reported error, compared with $4 \%$ of shifts $\geq 12.5$ h. ${ }^{16}{ }^{17}$

The lack of association between ICU experience and hypoglycaemia was surprising, as we expected that increased experience would reduce rates of medication errors. However, the beneficial effect of experience has been questioned in a number of studies. Previous studies that have demonstrated no significant relationship between self-reported errors and experience

Table 2 Conditional logistic regression analysis of the effect of work schedule on the risk for a hypoglycaemic event

\begin{tabular}{lll}
\hline & \multicolumn{2}{l}{ Per additional shift worked } \\
\cline { 2 - 3 } & OR $(\mathbf{9 5 \%}$ CI) & $\mathbf{p}$ Value \\
\hline Unadjusted & $1.56(0.98$ to 2.48$)$ & $\mathrm{p}=0.05$ \\
Adjusted & $1.65(1.01$ to 2.68$)$ & $\mathrm{p}=0.044$ \\
Adjustment variables & & \\
Nurse age (per year) & $1.02(0.97$ to 1.07$)$ & $\mathrm{p}=0.52$ \\
Nurse experience $(\geq 2$ vs $<2$ years $)$ & $1.13(0.47$ to 2.71$)$ & $\mathrm{p}=0.79$ \\
\hline
\end{tabular}


Table 3 Conditional logistic regression analysis of the effect of work schedule on the risk for a hypoglycaemic event stratified by error

\begin{tabular}{|c|c|c|c|c|}
\hline & \multicolumn{2}{|c|}{ Event associated with error $(\mathrm{N}=23)$} & \multicolumn{2}{|c|}{ Event not associated with error $(\mathrm{N}=18)$} \\
\hline & \multicolumn{2}{|c|}{ Per additional shift worked } & \multicolumn{2}{|c|}{ Per additional shift worked } \\
\hline & OR (95\% Cl) & p Value & OR (95\% Cl) & p Value \\
\hline Adjusted & $2.93(1.15$ to 7.44$)$ & $p=0.024$ & $1.34(0.73$ to 2.45$)$ & 0.34 \\
\hline \multicolumn{5}{|l|}{ Adjustment variables } \\
\hline Nurse age (per year) & $1.11(1.01$ to 1.23$)$ & $p=0.035$ & $0.97(0.89$ to 1.05$)$ & 0.39 \\
\hline Nurse experience ( $\geq 2$ vs $<2$ years) & $0.92(0.27$ to 3.21$)$ & $p=0.90$ & $1.71(0.42$ to 7.03$)$ & 0.46 \\
\hline
\end{tabular}

among nurses, ${ }^{8}$ less precise preparation of intravenous medications with increased years of experience ${ }^{14}$ and decreasing clinical performance with increased years of experience in physicians. ${ }^{18}$ One potential explanation is that recently hired nurses may be more vigilant early in their careers, and this may counterbalance any negative effects of inexperience.

One major strength of our study was the use of validated, as opposed to self-reported, safety outcomes and work schedules. However, we acknowledge that our study had a number of limitations. First, this study was conducted in only one ICU in a teaching hospital. These results may not be applicable to ICUs with different case mixes, algorithms for insulin/glucose management or commuting times. Second, we would not be able to generalise to ICUs where nurses work $8 \mathrm{~h}$ shifts. However, we doubt this is a major concern, as the vast majority of ICUs (88\% in a recent report ${ }^{19}$ ) schedule nurses based on $12 \mathrm{~h}$ shifts. Third, this study was not a prospective randomised trial, but was observational in nature; as such, there could still be residual confounding by other explanatory variables. Fourth, we studied only one ICU safety outcome, and we cannot extrapolate to other outcomes. Fifth, we only included control shifts before the hypoglycaemic event, as we were concerned that the occurrence of an event might have reduced the subsequent risk of hypoglycaemia due to increased vigilance on the part of nurses. Although we doubt it would have a substantial effect on the results, patients during the control period were earlier in their clinical course potentially introducing a bias into the study. Sixth, our ICU does not use mandatory overtime or traveling/ agency nurses and tends to have 1:1 nurse:patient ratios due to high patient acuity. Furthermore, very few of the nurses in our ICU work on other wards. Results from our ICU may not be generalisable to ICUs which do not have these characteristics.

\section{CONCLUSION}

In this preliminary single centre study, an increased number of shifts worked by the bedside nurse in the previous $72 \mathrm{~h}$ were significantly associated with hypoglycaemic events in critically ill patients who are receiving insulin infusions. The magnitude of the effect was greater in events associated with an error in management. This finding suggests that intensive nursing work schedules may compromise patient safety. Future studies may elucidate the optimal schedule to maximise patient safety, and whether work hour limits of nurses working in critical care units should be considered.

Acknowledgements We would like to thank A Maudee and K Nathoo, for their assistance and support with data collection, and M Brodie and M Maclure, for helpful reviews of the manuscript.

Funding This work was funded by a Michael Smith Foundation for Health Research (MSFHR) Infrastructure Grant (ICU Patient Safety). NTA was supported by a MSFHR Scholar Award and an Established Clinician Scientist Award from the Vancouver Coastal Health Research Institute. JMF was supported by a MSFHR Distinguished Scholar Award.

Competing interests None.
Ethics approval Ethics approval was provided by the University of British Columbia IRB

Contributors $\mathrm{KL}$ contributed to the design of the study, data acquisition, analysis and interpretation of the results. She drafted the original version of the manuscript and approved the final copy. RC contributed to the design of the study, data acquisition and interpretation of the results. She critically revised the manuscript and approved the final version. PD contributed to the design of the study, obtaining funding and interpretation of the results. He critically revised the manuscript and approved the final version. HW contributed to the design of the study, analysis of the data and interpretation of the results. He critically revised the manuscript and approved the final version. AW contributed to the design of the study, data acquisition and interpretation of the results. She critically revised the manuscript and approved the final version. MG contributed to the analysis of the data and interpretation of the results. She critically revised the manuscript and approved the final version. JMF contributed to the design of the study and interpretation of the results. He critically revised the manuscript and approved the final version. NA contributed to the design of the study, obtaining funding, data analysis and interpretation of the results. He was responsible for overall supervision of the study. He revised the manuscript and approved the final copy.

Provenance and peer review Not commissioned; externally peer reviewed.

\section{REFERENCES}

1. Cullen DJ, Sweitzer BJ, Bates DW, et al. Preventable adverse drug events in hospitalized patients: a comparative study of intensive care and general care units. Crit Care Med 1997:25:1289-97.

2. Rothschild JM, Landrigan CP, Cronin JW, et al. The critical care safety study: the incidence and nature of adverse events and serious medical errors in intensive care. Crit Care Med 2005;33:1694-700.

3. Bates DW. Unexpected hypoglycemia in a critically ill patient. Ann Intern Med 2002:137:110-16.

4. The NICE-SUGAR Study Investigators. Intensive versus conventional glucose control in critically ill patients. N Engl J Med 2009;360:1283-97.

5. Wiener RS, Wiener DC, Larson RJ. Benefits and risks of tight glucose control in critically ill adults a meta-analysis. JAMA 2008;300:933-44.

6. McCowen KC, Malhotra A, Bistrian BR. Stress-induced hyperglycemia. Crit Care Clin 2001;17:107-24.

7. Krinsley JS, Grover A. Severe hypoglycemia in critically ill patients: risk factors and outcomes. Crit Care Med 2007;35:2262-7.

8. Rogers AE, Hwang WT, Scott LD, et al. The working hours of hospital staff nurses and patient safety. Health Aff (Millwood) 2004;23:202-12.

9. Vriesendorp TM, DeVries JH, Hoekstra JBL. Hypoglycemia and strict glycemic control in critically ill patients. Curr Opin Crit Care 2008,14:397-402.

10. Breslow NE, Day NE. Statistical methods in cancer research. Volume 1: The Analysis of Case Control Studies. International Agency for Research on Cancer, 1980. 248-79

11. Van Dongen HP, Maislin G, Mullington JM, et al. The cumulative cost of additional wakefulness: dose-response effects on neurobehavioral functions and sleep physiology from chronic sleep restriction and total sleep deprivation. Sleep 2003;26:117-26.

12. Dawson D, Reid K. Fatigue, alcohol and performance impairment. Nature 1997;388:235.

13. Folkard S, Tucker P. Shift work, safety and productivity. Occup Med 2003:53:95-101.

14. Parsharum CS, To T, Seto W, et al. Systematic evaluation of errors occurring during the preparation of intravenous medication. Can Med Assoc J 2008;178:42-8.

15. Banks S, Dinges DF. Behavioral and physiological consequences of sleep restriction. J Clin Sleep Med 2007;3:519-28.

16. Scott LD, Rogers AE, Hwang WT, et al. Effects of critical care nurses' work hours on vigilance and patients' safety. Am J Crit Care 2006;15:1-8.

17. Balas MC, Scott LD, Rogers AE. Frequency and type of errors and near errors reported by critical care nurses. Can J Nurs Res 2006;38:24-41.

18. Choudhry NK, Fletcher RH, Soumerai SB. Systematic review: the relationship between clinical experience and quality of health care. Ann Intern Med 2005;142:260-73

19. Balas MC, Scott LD, Rogers AE. Frequency and type of errors and near errors reported by critical care nurses. Can J Nurs Res 2006;38:24-41. 